\title{
Model-Based Human Motion Tracking and Behavior Recognition Using Hierarchical Finite State Automata
}

\author{
Jihun Park ${ }^{1}$, Sunghun Park ${ }^{2}$, and J.K. Aggarwal ${ }^{3}$ \\ 1 Department of Computer Engineering \\ Hongik University, Seoul, Korea \\ jhpark@hongik.ac.kr \\ 2 Department of Management Information Systems \\ Myongji University, Seoul, Korea \\ shpark@mju.ac.kr \\ 3 Department of Electrical and Computer Engineering \\ The University of Texas at Austin, Austin, TX 78712 \\ aggarwaljk@mail.utexas.edu
}

\begin{abstract}
The generation of motion of an articulated body for computer animation is an expensive and time-consuming task. Recognition of human actions and interactions is important to video annotation, automated surveillance, and content-based video retrieval. This paper presents a new model-based human-intervention-free approach to articulated body motion tracking and recognition of human interaction using static-background monocular video sequences. This paper presents two major applications based on basic motion tracking: motion capture and human behavior recognition.

To determine a human body configuration in a scene, a 3D human body model is postulated and projected on a $2 \mathrm{D}$ projection plane to overlap with the foreground image silhouette. We convert the human model body overlapping problem into a parameter optimization problem to avoid the kinematic singularity problem. Unlike other methods, our body tracking does not need any user intervention. A cost function is used to estimate the degree of the overlapping between the foreground input image silhouette and a projected 3D model body silhouette. The configuration the best overlap with the foreground of the image least overlap with the background is sought. The overlapping is computed using computational geometry by converting a set of pixels from the image domain to a polygon in the $2 \mathrm{D}$ projection plane domain.

We recognize human interaction motion using hierarchical finite state automata (FA). The model motion data we get from tracking is analyzed to get various states and events in terms of feet, torso, and hands by a low-level behavior recognition model. The recognition model represents human behaviors as sequences of states that classify the configuration of individual body parts in space and time. To overcome the exponential growth of the number of states that usually occurs in a single-level FA, we present a new hierarchical FA that abstracts states and events from motion data at three levels: the low-level FA analyzes body parts only,
\end{abstract}


the middle-level FAs recognize motion and the high-level FAs analyze a human interaction. Motion tracking results and behavior recognition from video sequences are very encouraging.

\section{Introduction and Previous Work}

Analysis of video data is important due to the rapid increase in the volume of content recorded in the form of video. Recognition of human interaction in video is important to video annotation, automated surveillance, and contentbased video retrieval. Recognizing human interactions is a challenging task because it involves segmentation and tracking of articulated human body parts at low level and recognition of semantics in behavior at a higher level. This paper presents a model-based approach to motion tracking and recognition of human interaction in static-background monocular video sequences. Our motion tracking is based on computational geometry and forward kinematics to avoid the singularity problem [1], while our behavior recognition is based on hierarchical deterministic finite state automata (DFA) to abstract motion data in hierarchies. This paper is an extension to our previous papers[2,3,4]. This paper differs in hierarchically handling behavior recognition, and not relying on a distance map in overlapping computation, compensating recognition in [4].

While many others use stochastic sampling for model-based motion tracking, our method is purely dependent on parameter optimization. We convert the human motion-tracking problem into a parameter optimization problem. A cost function for parameter optimization is used to compute the degree of the overlapping between the foreground input image silhouette and a projected 3D model body silhouette. The overlapping is computed using computational geometry by converting a set of pixels from the image domain to a polygon in the real projection plane domain. One parameter optimization solves the model body configuration problem for a set of input images, and we compute the body configuration from each image frame. From a set of model body configuration data, we build the motion data. The model motion that we get from tracking is analyzed to get various states and events. From the motion data, we extract events that occur during the motion as well as changes in the configuration. We abstract numerical model body motion data into a sequence of state-change data. We recognize the motion of a single body from a sequence of state changes. We recognize interactive motion from each model body state changes. The above approach is hierarchical.

We may classify human motion analysis methods according to the recognition algorithms used: either stochastic algorithms such as hidden Markov models (HMM), or deterministic algorithms such as finite state automata (FA). If the uncertainty in image can be effectively resolved by model-based methods at the low level, then we can use deterministic methods for motion interaction recognition. In this case, the reliable overlapping between the model body and image data is useful. Many approaches have been proposed for behavior recognition using various methods, including HMM, FA, and context-free grammar. Oliver 
et al. [5] presented a coupled hidden Markov model for gross-level human interactions. Hongeng et al. [6] proposed probabilistic finite state automata(FA) for gross-level human interactions. Their system utilizes user-defined hierarchical multiple scenarios of human interaction. Hong et al. [7] proposed a DFA for detailed-level recognition of human gestures. Wada et al. [8] used nondeterministic finite state automata (NFA) using state product space. They preferred NFA to HMM [8] because NFA provides transparent state transition information whereas HMM's state transition is hidden to the user.

Our motion capturing and recognition framework successfully captures and recognizes five common human interactions: walking (i.e., approaching, departing), pushing, pointing, kicking, and handshaking. The major contributions of our work are as follows: (1) To overcome the occlusion problem, we have developed an overlapping area computation based on computational geometry, that automatically detects the initial configuration of the model body.[2,3] (2) To overcome the singularity problem encountered in inverse kinematics, we convert the problem to a forward kinematics based parameter optimization problem.[2] (3) Our motion tracking is fully automatic without any user intervention. (4) To overcome the problem of exponential growth of motion states encountered in single-level FA, we have developed a hierarchical FA to analyze body part motion and recognize motions and interactions. Motion capture results from color video sequences are very encouraging.

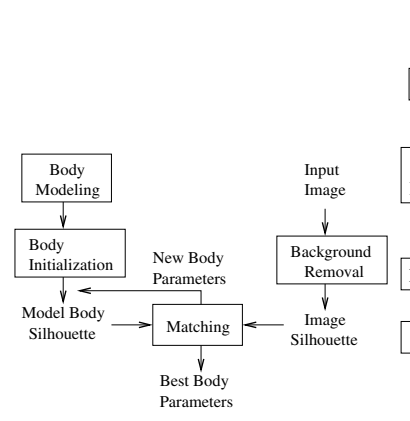

(a)

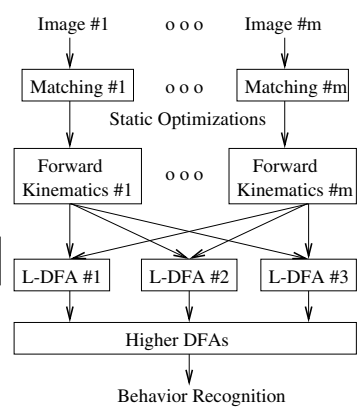

(b)

Fig. 1. Process of determining the best matching model body configuration for a single image(a), and a sequence of image matching, motion/behavior recognition(b).

\section{Overview of Our System}

Our system is designed for model-based human motion tracking and recognition of human interactions in surveillance videos. Figure 1(a) shows a matching process (i.e. computing the overlapping between the foreground input image and projected model body silhouette) given an input image. A 3D model body 
is built up. Model body joint angles and displacements are determined, and the body is projected on a $2 \mathrm{D}$ projection plane. On the $2 \mathrm{D}$ projection plane, we get a model body silhouette. The boxes in Figure 1 represent computational processes. The matching process uses static parameter optimization [9], which modifies the model body parameters and checks the resulting level of matching between the image silhouette and the model body silhouette for a single image. When the best matching model body configuration is found for a single image, then the process is done for that image; thus, for $n$ input images, we run the matching computation $n$ times to get $n$ set of tracked motion data.

Figure 1(b) shows the sequence of matching process tasks. When the matching computation is completed using static optimization, we have a model body configuration for each image. Then we run forward kinematics to determine the kinematic parameters such as hand position and foot position for each image. The kinematic parameters of the fitted model body form the motion data, which is then analyzed by a recognition model. We propose a hierarchical deterministic finite state automata (DFA) as the recognition model. The hierarchical DFA is composed of low-level DFAs to abstract numerical motion data and analyze the motion data with respect to the feet, torso, and hands. The low-level DFAs independently represent the individual body-part poses as discrete states and the body part motion as transitions between the states. The body part motion recognition results from the low-level DFA are fed into middle-level DFAs for the recognition of a whole body motion, and then fed into higher-level DFAs to analyze the interactive motion behavior between two persons.

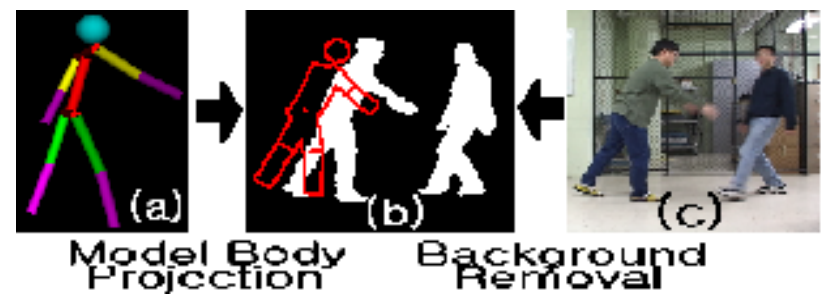

Fig. 2. 3D Model body(a), overlapping between background removed image and projected model body(b), and input image(c)

In this section, we present our optimization cost function to find the best overlap. The main cost function is very similar to our previous functions $[2,3$, 4] except that we no longer use a distance map. As shown in Figure 2(a), the body is modeled as a configuration of nine cylinders and one sphere. These are projected onto a $2 \mathrm{D}$ real projection plane. A sphere represents the head, while the rest of the model body is modeled using cylinders of various radii and lengths. Currently, we use only nine 1-DOF (1 degree-of-freedom) joints plus body displacement DOF. These are our control variables for the parameter optimization and the cost function. 


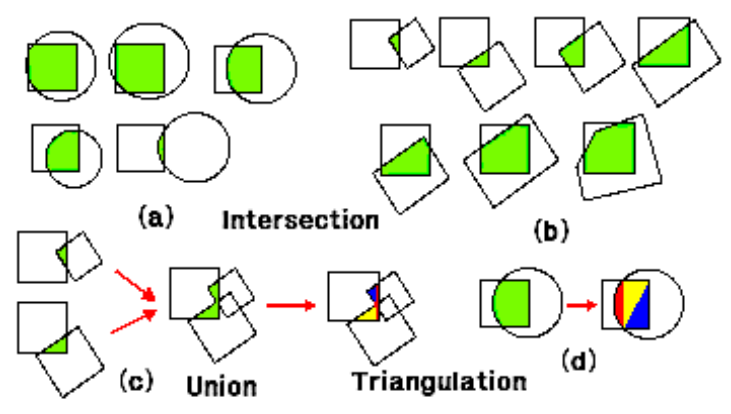

Fig. 3. Five possible cases of a pixel(square) partially occluded by a model head(a), seven possible cases of a pixel(square) partially occluded by a polygon body(b), union of intersected area, then triangulation for a polygon body(c), and union and intersection computation for a model head(d)

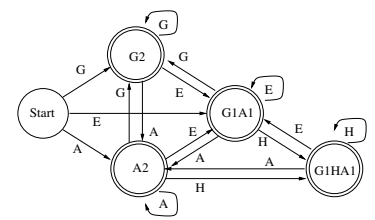

(a) Foot movement analysis.

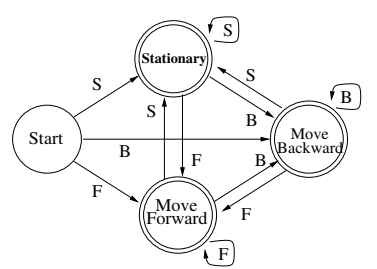

(b) Body center movement analysis.

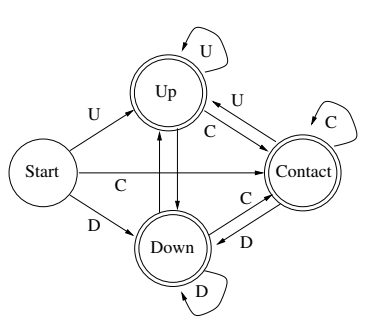

(c) Hand movement analysis.

Fig. 4. Lower level finite state automatas for recognizing body part motion.

While a model silhouette is computed by a 3D human model projection, an image silhouette has been converted from the 2D integer pixel domain to a real domain such that the resulting image silhouette becomes a jagged-edge polygon with only horizontal and vertical edges. We compute the polygon intersection between the image silhouette and the model silhouette. We found the best fitting configuration using the GRG2[9] optimization package with the best matching overlap between the model body silhouette and the foreground of the input image. Figure 2(b) shows the initial state of searching for the best overlapping configuration, given the first frame image of the video sequence of Figure 6(e). As can be seen in Figure 2(b), the initial joint angle values of the model body for parameter optimization are arbitrary. This shows that our initial model body configuration detection for the first image frame is automatic. We know the center of the foreground of an input image, and matching is done using optimization. We know by how much the model part covers/misses the foreground/background of an input image. From the foreground image, we can compute how tall and thick the human is because we are given side view of the input images. By finding the best overlapping, we automatically find the best body configuration. The background removal process is presented in our previous papers[2,3,4]. 
Figure 3 shows 12 possible overlapping cases in which either a model head or polygon-shaped body, generated after 3D model body projection, is overlapping with a pixel. In the figure, a circle represents a projected head outline, and an irregular polygon represents a body part, while a square represents a pixel. The union of these irregular-shaped objects results in a projected model body silhouette. After union computation, a triangulation process is needed to compute the area of the unioned irregular shaped object. Because our cost function of the parameter optimization works only in the real number domain, we cannot work on a purely pixel-based integer number domain cost function. Thus we have to compute the pixel overlapping area to eliminate an integer cost function.

\section{Hierarchical Deterministic Finite State Automata for Behavior Recognition}

We model human behavior as sequences of state changes that represent the configuration and movement of individual body parts (i.e., legs, torso, and hands) in spatio-temporal space. We employ a sequence analyzer and an event detector that are quite similar to those of [8]. However, our sequence analyzer is a DFA that tracks status changes, unlike the nondeterministic finite state automata of [8], while the event detector allows state transition. Our DFAs are quite unique because we have hierarchical layers of sequence analysis. The use of hierarchical DFAs reduces the exponentially large number of states to be handled in behavior recognition. Each DFA consists of a finite set of $\operatorname{states}(Q)$, an initial state $\left(q^{0}\right)$, a finite set of events $\left(\sum\right)$, a state transition function $(\delta)$, and a finite set of final states $(F)$. It is represented by $\left(Q, q^{0}, \sum, \delta, F\right)$. Each state $q^{i}$ in the sequence $\left(q^{0}, q^{1}, \cdots, q^{n}\right)$ corresponds to a frame. To handle every possible situation, our low-level sequence analyzers are of the form $\left({ }_{m}^{p} Q,{ }_{m}^{p} q^{0},{ }_{m}^{p} \sum,{ }_{m}^{p} \delta,{ }_{m}^{p} F\right)$, where $p, p=1,2,3$, is an index for body parts, index number one for body center, index number two for feet, index number three for hands, and $m$ is an index for each person in the scene. ${ }_{2}^{1} q^{i} \in{ }_{2}^{1} Q$ means ${ }_{2}^{1} q^{i}$ is a state of sequence index number $i$, of a second person in the scene, of body part index one, the body center. The event detector detects events or status changes while reading model body motion data obtained from a sequence of parameter optimization. Events are determined from model motion data. To detect a specific event, it is necessary to check a sequence of motion.

We employ DFAs on three levels: A separate low-level DFA is employed for each body part: body center (torso), feet, and hands. Each low-level DFA considers all possible states for its body part (hand, torso or feet), independent of the rest of the body. Low-level DFA input is a set of numerical data that is abstracted and converted into a sequence of status changes. We allow only four states for feet: both feet on the ground, both feet in the air, one foot in the air while the other is on the ground, and one foot in the high air while the other is on the ground. (This state is to recognize violent foot motion.) The walking motion is less dependent on arms or hand movement. Figure 4(a) shows a DFA to analyze feet status. At the start state, there are only four transitions possible 
because we classify every situation into one of four states. State G2 means a status in which both feet are on the ground, state A2 means both feet are in the air, state G1A1 means one foot is on the ground while the other is in the air, and state G1HA1 means one foot is in the high air. The state transition occurs when a condition in the motion configuration is satisfied, and is denoted as G, A, E, or H. Similarly, we define three body center (torso) states: stationary, moving forward, and moving backward, denoted as S, F, and B, respectively. Figure 4(b) shows a DFA to analyze body center status. Three states are defined for hands: both hands down (D), at least one hand raised (U), and at least one hand in contact with another person (C). Figure 4(c) shows a DFA to analyze the status of the hands. We do not differentiate between hands.

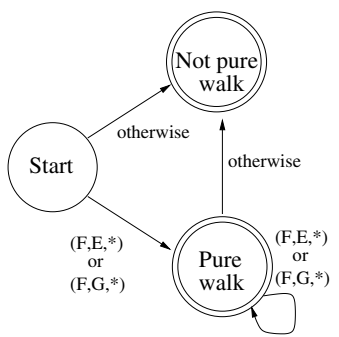

(a) Recognizing pure walking

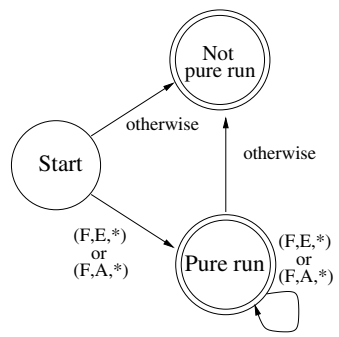

(b) Recognizing pure running

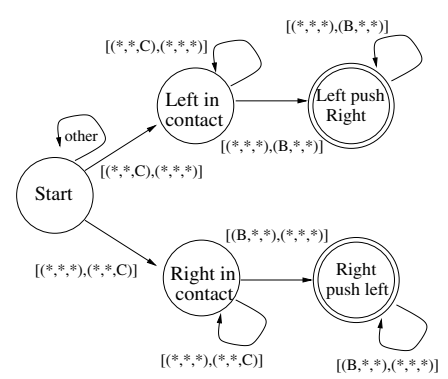

(c) High-level DFA.

Fig. 5. Middle-level finite state automatas for recognizing motion of a single person(a,b), and high-level DFA for recognizing interactive (pushing) motion of two per$\operatorname{sons}(\mathrm{c})$.

A middle-level DFA that takes low level DFA state changes as input is used to recognize a single body. Figure $5(\mathrm{a}, \mathrm{b})$ shows middle-level DFAs for recognizing walking and running. We consider a tuple of states for a middle-level DFA, $\left({ }_{m}^{1} q^{i},{ }_{m}^{2} q^{i},{ }_{m}^{3} q^{i}\right)$, a token made of low-level state transitions of a model body of index number $m$, to recognize its motion. ${ }^{*}$ means any possible input. Each DFA recognizes a specific motion only. DFAs can be modified for user's video content retrieval request. State changes at low level DFA are fed into a middle level DFA, which determines a single body motion status. The results from the middle-level DFA is a higher-level motion description such as "stand still" or "kick" of a single person. The outputs from the middle-level DFA as well as low-level DFAs are fed into the high-level DFA, which analyzes the interactive motion behavior between model bodies.

We now explain how the high-level DFA works. To recognize an interactive motion between two persons, we need a tuple of states, $\left[\left({ }_{1}^{1} q^{i},{ }_{1}^{2} q^{i},{ }_{1}^{3} q^{i}\right),\left({ }_{2}^{1} q^{i},{ }_{2}^{2} q^{i},{ }_{2}^{3} q^{i}\right)\right]$. The tuple consists of states of a left and right person's body part status. The higher-level DFA(s) states recognize behavior based on lower-level sequence analyzers, thirteen lower-level states to abstract motion data. Rather than using an exponentially increasing number of entire states, we 
focus on a subset of all states. Each tuple we fed into a higher-level DFA corresponds to one of an exponentially large number of states. For a higher-level DFA, the number of states can be relatively small. For each person in a scene, there are approximately 36 possible states because we use three or four states for each of the three body parts. If there are two persons involved in an interaction, we would need to make a DFA of minimum 1296 states to handle all possible motion states. Generally, we need $\left|{ }_{1}^{1} Q\right| \times\left|{ }_{1}^{2} Q\right| \times\left|{ }_{1}^{3} Q\right| \times\left|{ }_{2}^{1} Q\right| \times\left|{ }_{2}^{2} Q\right| \times\left|{ }_{2}^{3} Q\right|$ states for an interaction of two persons, where $|Q|$ is the number of states in $Q$. It is plain that this exponential growth will quickly become intractable. Rather than generating 1,296 states and designing state transitions, we design three or four states to recognize each motion of a body part, totaling 13 states for any number of persons. As a result, we need to design a higher level DFA to recognize behavior based on lower-level sequence analyzers, plus 13 lower-level states to abstract motion data rather than 1,296 states and state transition designs.

\section{Experimental Results}

Our system was used to recognize five 2D-based human motions: walking (i.e., approaching, departing), pushing, kicking, pointing, and handshaking. Figure $6(\mathrm{f}, \mathrm{h})$ shows two persons shaking hands in front of a static background. The red line shows the union of every model body part. As long as there is no heavy occlusion between the subjects, motion tracking is satisfactory because of geometric union computation in handling occlusion. The motion tracking is excellent, as shown in Figure 6. Figure 6(g,i) shows a walking (departing) motion. After motion tracking, we get two sets of motion data, one for each person appearing in the scene. The raw motion data is abstracted and converted to a sequence of states for each body part.

Figure 6(a) shows a pushing motion. The right person approaches and pushes the left person. As a result of being pushed, the left person moves backward. This is an example of a scene in which a cause (pushing) of a person resulted in a change (move backward) in the other person. This interaction can be only recognized by checking all 1296 states, including many states that are not directly related to a pushing motion. SsSSSSSSSSSSSNNSSNNNNNNNNNNNNNNNNNNNN is a sequence of body center states of the left person in the scene, where $\mathbf{S}$ represents "stand still" state, and $\mathbf{N}$ means "move negative." DuuuucccccccuUUUDDDDDDDDDDDDDDDDDDDDD is a sequence of hand states of the right person in the scene, where $\mathbf{D}$ represents "the both hands down" state, U means "any hand up", and C means "hand(s) in contact." Two sequences of the input tuple consist of these state change sequences. From the input tuple, we can easily recognize behavior: "right person contacted the left person, and the left person has moved to negative direction," meaning the right person pushed away from the left person. We need at least four states to recognize a pushing motion: one representing the contact state by a pushing person, and the other representing the backwards movement of the pushed person, for both persons in the scene. A complicated query is of the form "a person approached the other, and pushed away," which would require all three levels of DFA-based sequence analysis. 
(a)
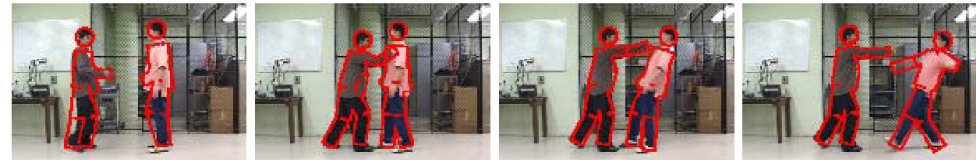

(b)
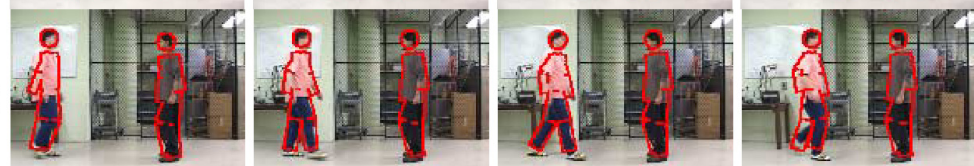

(c)
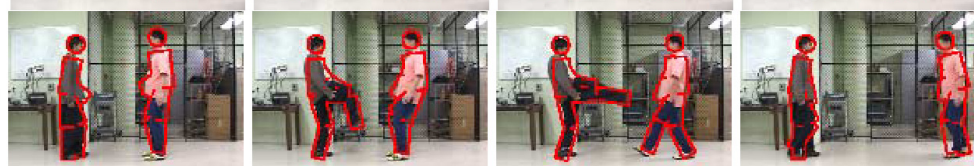

(d)
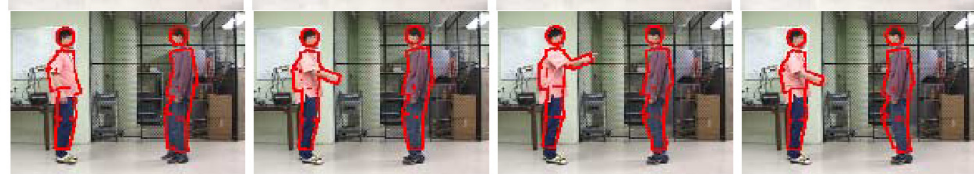

(e)
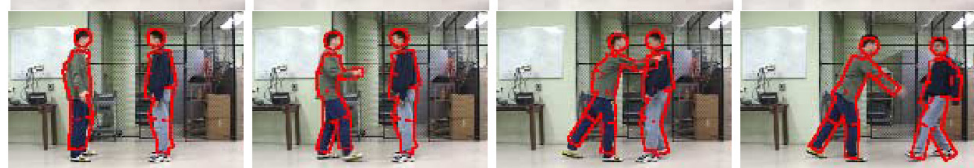

(f)
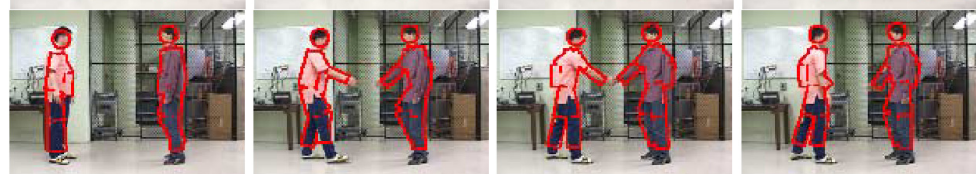

(g)
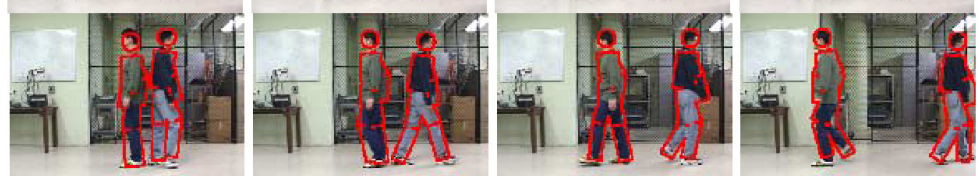

(h)
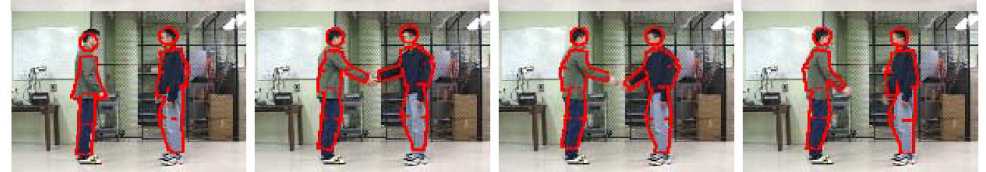

(i)
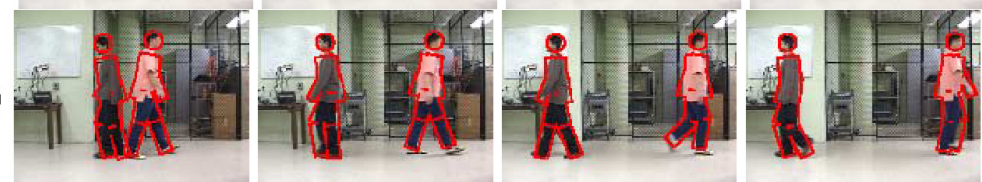

Fig. 6. The subject, with the model figure superimposed, shown over a pushing motion (a), a walking (approaching) motion (b), a kicking motion (c), a pointing motion (d), a pushing motion (e), a hand-shaking motion (f), a walking (departing) motion (g), a hand-shaking motion (h), and a walking (departing) motion (i). 


\section{Conclusion}

In this paper, we presented a new approach to human motion capture and its behavior analysis using hierarchical DFAs. The model based-method at the image processing level uses a 3D human body model and parameter optimization techniques to achieve refined segmentation and tracking of the moving humans. Use of the model body in human motion tracking allows us to take advantage of the knowledge of the human body inheritance in the model, making the system more robust. The motion tracking results from video sequences are very encouraging, although it performs best on side views. The output data from model-based human tracking enables us to recognize human behavior in the input scene. Rather than using an exponentially increasing number of entire states, we focus on a subset of all states. Our recognition framework successfully recognizes various human interactions between two persons, although our current motion states cannot cover all human motion.

Acknowledgements. This research was supported by the 2004 Hongik University Academic Research Support Fund. We thank Ms. Debi Prather for proofreading of this paper.

\section{References}

1. Morris, D., Rehg, J.: Singularity analysis for articulated object tracking. In: Computer Vision and Pattern Recognition. (1998)

2. Park, J., Park, S., Aggarwal, J.K.: Human motion tracking by combining viewbased and model-based methods for monocular video sequences. Lecture Notes in Computer Science (2003 International Conference on Computational Science and Its Applications) 2669 (2003)

3. Park, J., Park, S., Aggarwal, J.K.: Model-based human motion capture from monocular video sequences. Lecture Notes in Computer Science (ISCIS 2003) 2869 (2003)

4. Park, S., Park, J., Aggarwal, J.K.: Video retrieval of human interactions using model-based motion tracking and multi-layer finite state automata. Lecture Notes in Computer Science (2003 Intl. Conf. on Image and Video Retrieval) 2728 (2003)

5. Oliver, N.M., Rosario, B., Pentland, A.P.: A Bayesian computer vision system for modeling human interactions. IEEE Trans. Pattern Analysis and Machine Intelligence 22 (2000) 831-843

6. Hongeng, S., Bremond, F., Nevatia, R.: Representation and optimal recognition of human activities. In: IEEE Conf. on Computer Vision and Pattern Recognition. Volume 1. (2000) 818-825

7. Hong, P., Turk, M., Huang, T.S.: Gesture modeling and recognition using finite state machines. In: IEEE Conf. on Face and Gesture Recognition. (2000)

8. Wada, T., Matsuyama, T.: Appearance based behavior recognition by event driven slective attention. In: IEEE Computer Society Conference on Computer Vision and Pattern Recognition, Orlando, FL (1998) 759-764

9. Lasdon, L., Waren, A.: GRG2 User's Guide. (1989) 